Plot a curve with $y=\lambda^{2}$ and $x=l . \quad V$ is known from the curve for volumes; then $F_{1}-F_{0}=\pi^{2} / V^{2}$ into the area under the curve between the limits $l_{1}$ and $l_{0}$.

This theory can evidently be extended to calibration for other functions of the radius.

In practice it is advisable to run the thread through the tube more than once, as the points on the curve of one run may not exactly coincide with those for another.

\title{
The Production of Ozone by a Photo-Electric Current in OXYGEN. ${ }^{1}$
}

By Harry S. Hower.

C LTRA-VIOLET light was allowed to fall upon a polished platinum knob in an oxygen atmosphere.

The knob being changed to about $-1,500$ volts, the discharge current from it to the earthed wires opposite it was measured by a galvanometer in series. The ozone produced was detected by its effect on a piece of platinum foil. The foil changes its position in the voltaic series when it absorbs $\mathrm{O}_{3}$.

\section{Note on Electric Double Refraction in Carbon-Disulphide at Higher Temperatures. ${ }^{1}$}

By L. B. MoRse.

\begin{abstract}
A $\mathrm{T}$ a former meeting of the Physical Society the late D. B. Brace reA ported that he had not been able to observe the "Kerr effect" in air. A beam of plane polarized light showed no trace of ellipticity after passing back and forth several times in the air between long charged plates.

Schmidt's curve, for the effect of temperature on the Kerr effect in $\mathrm{CS}_{2}$, shows a more rapid rate of decrease of the effect with increase in temperature, at the higher than at the lower temperatures at which he worked.

At the time this work was begun an apparatus was being constructed in which ordinary gases, gaseous $\mathrm{CS}_{2}$, etc., were to be tested under varying conditions of temperature and pressure.

In view of the above and other considerations it seemed important to

${ }^{1}$ Abstract of a paper presented at the Ithaca meeting of the Physical Society, June 29July 3, 1906 .
\end{abstract}

